\title{
Absence of localization and large dc conductance in random superlattices with correlated disorder
}

\author{
Enrique Diez and Angel Sánchez \\ Escuela Politécnica Superior, Universidad Carlos III de Madrid, C. Butarque, 15, E-28911 Leganés, Madrid, Spain \\ Francisco Domínguez-Adame \\ Departamento de Física de Materiales, Facultad de Físicas, Universidad Complutense, E-28040 Madrid, Spain
}

(Received 17 June 1994)

\begin{abstract}
We study how the influence of structural correlations in disordered systems manifests itself in experimentally measurable magnitudes, focusing on dc conductance of semiconductor superlattices with general potential profiles. We show that the existence of bands of extended states in these structures gives rise to very noticeable peaks in the finite-temperature dc conductance as the chemical potential is moved through the bands or as the temperature is increased from zero. On the basis of these results we discuss how dc conductance measurements can provide information on the location and width of the bands of extended states. Our predictions can be used to demonstrate experimentally that structural correlations inhibit the localization effects of disorder.
\end{abstract}

\section{INTRODUCTION}

A number of recent papers have proposed and provided theoretical evidence that in disordered systems where the disorder exhibits some kind of short-range spatial correlation, wave localization may be inhibited and bands of extended states appear. ${ }^{1-18}$ This phenomenon has been shown to arise in a number of different contexts, like electron transport, ${ }^{1-9,12-14}$ phonon transport, ${ }^{11,17}$ exciton dynamics, ${ }^{15,16,18}$ or magnon propagation. ${ }^{10}$ All of these theoretical analyses openly contradict the belief that localization of all eigenstates is a general phenomenon in one-dimensional disordered systems. Note, however, that this belief has only been rigorously proven for some uncorrelated random systems, ${ }^{19}$ and hence the existing theorems do not apply to the above cases. In spite of this, there is some controversy as to the relevance of these results and the nature of the band or bands of extended states, and delocalization by structural correlation is still not generally accepted. Therefore, we undertook the task of finding experimentally measurable quantities and physically realizable systems that allow for a clearcut validation of the above mentioned results. We have already proposed experiments on mechanical models, ${ }^{11,17}$ but, admittedly, these may be hard to construct and seem rather artificial or academic systems. For this reason, we decided to concern ourselves with a more interesting system, namely, semiconductor superlattices.

Nonperiodic (quasiperiodic or disordered) semiconductor superlattices are being studied with increasing interest in the last decade. First, Merlin and co-workers studied Fibonacci superlattices ${ }^{20}$ where the unusual, fractal-like spectral properties give rise to very characteristic properties. Shortly thereafter, localization was observed in intentionally disordered $\mathrm{GaAs} / \mathrm{Ga}_{1-x} \mathrm{Al}_{x} \mathrm{As}$ superlattices. ${ }^{21}$ This was followed by a number of studies on disordered superlattices, ${ }^{22}$ that showed a much larger photoluminiscence intensity than ordered lattices $^{23}$ among other different features that we do not describe here. ${ }^{24,25}$ Other materials like $\mathrm{Si}_{1-x} \mathrm{Ge}_{x} / \mathrm{Si}$ have been shown to exhibit the same phenomenon. ${ }^{26}$ Thus, this rather good knowledge already available as well as recent advances of molecular beam expitaxy make these systems the ideal candidates to propose experiments on localization or delocalization electronic properties.

The paper is organized as follows. In Sec. II, we present our model and summarize previous work by us ${ }^{13,14}$ which is necessary for a better understanding of the present paper. The body of the paper is Sec. III, where we present our results on finite-temperature dc conductance. We begin by discussing the motivation of the calculation. Then, we proceed on to zero-temperature dc conductance which is mainly determined by the transmission coefficient. Most of the section is devoted to finite-temperature dc conductance. We describe the dependence of this magnitude on the chemical potential of the sample and on the temperature; besides, we also study how the conductance relates to the model parameters. We show how the bands of extended states reveal themselves through a well-defined peak in the dc conductance. In addition, we also study the high temperature limit where we find a power-law scaling of the conductance with the system size. Finally, in Sec. IV, we discuss our results and how they can be related to actual measurements to infer the main characteristics of the bands of extended states from experiments on superlattices.

\section{MODEL AND BACKGROUND}

\section{A. The Kronig-Penney model and its application to superlattices}

The basis of our model is the Kronig-Penney ${ }^{27}$ one, in which it is assumed that the electron interaction with the 
underlying one-dimensional lattice is given by a potential of the form

$$
V(x)=\sum_{n} \lambda_{n} \delta\left(x-x_{n}\right)
$$

This model is very general, and aside from the application to disordered semiconductor superlattices we are going to describe, it also appears in many other contexts like other microelectronic devices, ${ }^{28}$ localization phenomena in liquids, ${ }^{29}$ physical properties of layered superconductors, ${ }^{30}$ and quark tunneling in onedimensional nuclear models ${ }^{31}$ to name a few. Regarding superlattices, the choice of potential $V(x)$ given by Eq. (1) is very general as, in principle, the superlattice potential could take many different shapes: Square barriers, $V$-shaped wells, sawtooth, parabolic, etc. In fact, what we are doing is assuming an expression for the cell potentials in terms of point interaction potentials. The term point interaction refers to any arbitrary sharply peaked potential approaching the $\delta$ function limit (zero width and constant area). Such potentials are often used in a variety of physical contexts in solid state physics, ${ }^{32}$ since, with limitations, they are good candidates to replace actual, short-ranged, one-dimensional potentials. ${ }^{33}$ Moreover, it has been recently demonstrated that the discretized form of the Schrödinger equation for an arbitrary potential in one dimension can be mapped onto a Kronig-Penney model. ${ }^{34}$ Hence, the use of potential (1) is not a serious restriction to simulate actual semiconductor superlattice potentials within the envelope-function formalism.

\section{B. The continuous random dimer model}

The version of the Kronig-Penney model we are interested in is the so-called continuous random dimer model (CRDM), which was first introduced by us in Refs. 13 and 14 as a realistic theoretical scenario where delocalization effects have dramatic consequences. We note that the model is inspired by the tight-binding random dimer model of Dunlap et al., ${ }^{2,5}$ and that in view of their results the essential physics of our model is qualitatively the same as regards the existence of a nonzero measure set of extended states. There are two different features of our model: First, as we will show below, it exhibits an infinite number of bands of extended states, which makes it interesting from the view point of applications as there are many options to match the Fermi level; and second, the fact that our model is continuous and includes backscattering effects gives it a more realistic character and hence it strongly supports the possibility of seeing these effects in a variety of actual physical systems. Our model is defined by particularizing Eq. (1) as follows: First, we choose $\lambda_{n}>0$; the extension of the results to the $\lambda_{n}<0$ case is straightforward, although the choice of the sign is irrelevant for the superlattice application as $\lambda_{n}$ may be always taken as positive by a suitable assignation of the $\delta$ function to superlattice blocks. Second, we take the positions of the $\delta$ potentials to be regularly spaced, i.e., $x_{n}=n$. Third and most important, we introduce a paired correlated disorder which implies that $\lambda_{n}$ takes only on two values, $\lambda$ and $\lambda^{\prime}$, with the additional constraint that $\lambda^{\prime}$ appears only in pairs of neighboring sites (dimer). The corresponding Schrödinger equation is then (we use units such that $\hbar=2 m=1$ in the rest of the paper; energy, $k T$ and the chemical potential below will be measured accordingly)

$$
\left[-\frac{d^{2}}{d x^{2}}+\sum_{n} \lambda_{n} \delta(x-n)\right] \psi(x)=E \psi(x) .
$$

In Ref. 14 we developed a generalized Poincaré map formalism that allows to map general one-dimensional Schrödinger equations onto discrete equations exactly, for any potential allowed in quantum mechanics. In particular, its application to Eq. (2) is quite simple. For the sake of brevity, we only quote here the final result, and refer the reader to Sec. II of Ref. 14 for the details. Equation (2) is exactly equivalent to

$$
\psi_{n+1}+\psi_{n-1}=\left[2 \cos q+\frac{\lambda_{n}}{q} \sin q\right] \psi_{n},
$$

where we have put $\psi_{n} \equiv \psi(x=n)$ and $q \equiv \sqrt{E}$.

From the above equation, we can see that there are an infinite number of resonant energies for which the reflection coefficient of a single dimer vanishes. Indeed, taking into account that the condition for an electron to move in the perfect lattice $\left[\lambda_{n}=\lambda\right.$ for all $n$ in Eq. (3)] is

$$
\left|\cos q+\frac{\lambda}{2 q} \sin q\right| \leq 1
$$

gives us the first restriction on the allowed values of energy. Further, introducing a single dimer occupying sites $n=0$ and 1 and eliminating $\psi_{0}$ and $\psi_{1}$, we obtain

$$
-\psi_{2}=\left(\omega+\omega^{\prime}-\omega^{\prime 2} \omega\right) \psi_{-1}-\left(1-\omega^{\prime 2}\right) \psi_{-2},
$$

with $\omega \equiv 2 \cos q+(\lambda / q) \sin q$ and $\omega^{\prime}$ the same exchanging $\lambda$ by $\lambda^{\prime}$. It is evident from this expression that if $\omega^{\prime}=0$, we recover the equation for the perfect lattice with sites $n=0$ and 1 suppressed except for an irrelevant phase factor $\pi$. This means that at the particular values $q_{r}$ such that $\omega^{\prime}=0$, the reflection coefficient of the dimer vanishes. Such a condition and the perfect lattice one (4) yield the two equations determining the resonances $E_{r}=q_{r}^{2}$,

$$
\begin{aligned}
\left|\cos q_{r}\right| & \leq \frac{\lambda^{\prime}}{\left|\lambda-\lambda^{\prime}\right|} \\
-\frac{2}{\lambda^{\prime}} & =\frac{\tan q_{r}}{q_{r}}
\end{aligned}
$$

which is our final result. Restricting ourselves to the range $\lambda \leq 2 \lambda^{\prime}$ Eq. (6a) is trivially satisfied. Then, Eq. (6b) has an infinite number of solutions, one in every interval $[(2 n-1) \pi / 2,(2 n+1) \pi / 2], n=1,2, \ldots$ leading to infinite energy values for which the reflection coefficient of a single dimer vanishes. 


\section{Properties of the model}

Of course, the above result does not imply anything about extended states in a CRDM with a finite density of dimers, and it is necessary to study that problem separately. This we carried out in Refs. 13 and 14 by means of numerical evaluation of exact expressions obtained via transfer matrix techniques for the relevant quantities: Transmission coefficient, Landauer resistance, Lyapunov coefficient, and density of states. The behavior of all these quantities, combined with multifractal and inverse participation ratio analyses, allowed us to establish on firm grounds that the single dimer resonances survive in the CRDM and, moreover, that they give rise to bands of finite width of truly extended states. The interested reader may find a thorough report in Ref. 14. Here, we will only comment on one of these magnitudes, namely, the transmission coefficient, which is the starting point for our computations of finite-temperature dc conductance.

An example of the behavior of the transmission coefficient around one of the resonant energies is shown in Fig. 1 for a dimer concentration $c=0.5$ ( $c$ is defined as the ratio between the number of $\lambda^{\prime}$ and the total number of $\delta$ 's in the lattice). We stress that, in spite of the fact that the plot corresponds to an average over 100 realizations of the CRDM, the transmission coefficient for typical realizations behaves in the same way, although noisier. Thus, the only effect of averaging is to smooth out particular features of realizations keeping only the main common characteristic, i.e., the wide transmission peak. This is the property we want to highlight: Close to single dimer resonances (in the case of Fig. 1, the first one, which occurs at $E_{r}=3.7626 \ldots$ for the chosen parameters $\lambda=1.0, \lambda^{\prime}=1.5$ ), there is an interval of energies that shows also very good transmission properties, similar to those of the resonant energy. Most important,

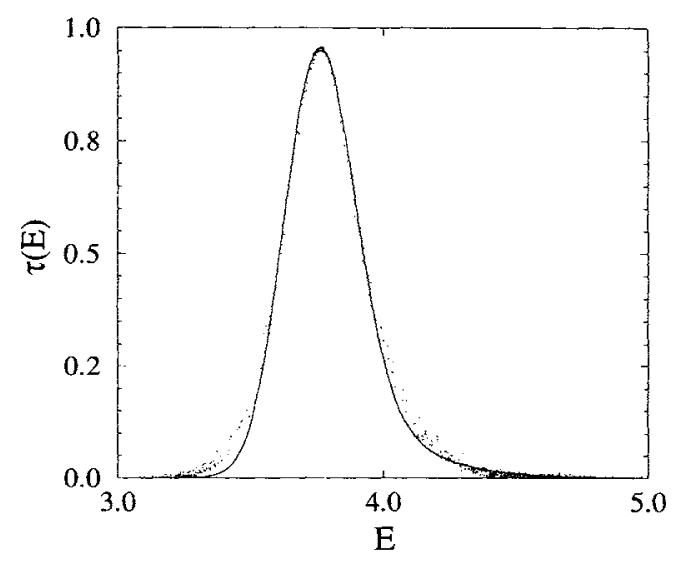

FIG. 1. Transmission coefficient for the CRDM with a dimer concentration $c=0.5$. The $\delta$ function strengths are $\lambda=1, \lambda^{\prime}=1.5$. Shown is an average over 100 realizations. Every realization consists of 15000 scatterers. The first allowed band in the perfect lattice is $(0.921,9.870)$. The solid line is an analytical fit using Moyal and Gaussian functions (see text for details). such an interval has always had a finite width, for all values of dimer concentration, $\lambda$ and $\lambda^{\prime}$ (provided they satisfy the above conditions), or number of sites in the lattice. The peak width depends on the order of the resonance (the higher the resonance the wider the band of states with transmission coefficient close to unity) and the concentration of dimers (the larger the concentration, the narrower the peak, being always of finite width as already stated). Figure 1 also shows an analytical fit to the shape of the transmission coefficient dependence on the energy, which will be used below. The parametrization used in the best fit close to $E_{r}$ is $\tau(E)=m(E)+g(E)$, where

$$
m(E)=m_{0} \exp \left(-\frac{v+e^{-v}}{2}\right)
$$

is the Moyal ${ }^{35}$ function with $v=\left(E-E_{r}\right) / s$, and

$$
g(E)=g_{0} \exp \left(-\frac{\left(E-E_{r}\right)^{2}}{2 \sigma^{2}}\right)
$$

is the usual Gaussian function. The parameters that fit data in Fig. 1 are $m_{0}=0.65, s=0.16, g_{0}=0.70$, and $\sigma=$ 0.26 . As can be seen from Fig. 1, the fitting reflects the asymmetry of the peak and corresponds very well to the average transmission coefficient. Of the above constants, the most relevant one is probably the variance of the gaussian; we will come back to this parameter and its relevance below.

\section{FINITE-TEMPERATURE dC CONDUCTANCE}

\section{A. Motivation: Characteristics to determine}

So far, we have summarized the main properties of the CRDM, which can be found in full detail in Ref. 14. The crucial conclusion of those previous studies has been already mentioned: There are bands (an infinite number of them) of truly extended states in the CRDM in spite of the disorder. We have provided enough theoretical evidence and we can be quite sure of the correctness of that statement. The most important point, however, regards applications of this result, and this inmediately implies two questions: First, are these bands of extended states experimentally measurable? Admittedly, if the extended states we have predicted are not seen in actual physical systems, the question as to their true extended nature becomes irrelevant. Second, do these extended states serve as the basis for new applications or devices? Hopefully, the answer to this second question would be yes provided the answer to the first question was also yes. This may be easily understood if we think that the transport properties of such microstructures would depend strongly on the value of the incoming energy, and therefore they could serve as filters of unwanted energies (in fact, we have proposed similar applications in mechanical devices based on the same ideas; ${ }^{17}$ we will discuss this application later on in the conclusion section). It is then clear than 
the crux of the problem is the first question, and that would be the one we will try to answer in the remainder of the paper. Specifically, we will devote ourselves to show how the position of the peak and its width may be determined from finite-temperature dc conductance measurements, and how experiments relate to the dimer concentration.

\section{B. Zero-temperature dc conductance}

In this subsection, we discuss electron propagation at very low temperatures through a disordered superlattice with one of the two alternating types of constituents subject to the constraint of the CRDM. We note at this point that the $\delta$ function may represent the joint potential of several layers, e.g., a block GaAs- $\mathrm{Ga}_{1-x} \mathrm{Al}_{x} \mathrm{As}-\mathrm{GaAs}$ giving rise to a square potential barrier. Different choices of the blocks are then associated to the two different values of $\lambda$ and $\lambda^{\prime}$. We will term these superlattices correlated disordered superlattices (CDSL). In general, two main factors must be taken into account when dealing with vertical transport through a CDSL. On the one hand, since this is essentially a quantum phenomenon, we must consider systems with strong coupling between adjacent blocks, but in our model this is not a problem since the distance between $\delta$ functions does not play any role aside from fixing the resonant energies. On the other hand, we are neglecting electron-phonon scattering effects which tend to disrupt coherent quantum transport. These effects crucially depend on the sample temperature, so it may be confidently expected that their influence can be neglected at very low temperatures. Besides, superlattices used for experiments have periods in the range from one monolayer to several nanometers. ${ }^{21-26}$ A short spacing between layers also contributes to reduce the scattering by phonons, and therefore, short-period superlattices could be useful for the work we propose up to higher temperatures, because as we have seen the period length is not very relevant. Hence, a physical realization of our model is possible and the measurements should be comparable to our predictions in a wide range of temperatures.

The electrical conductance at zero temperature can be obtained straightforwardly from the well-known dimensionless single-channel Landauer formula ${ }^{36}$

$$
\kappa_{0}(E)=\frac{\tau(E)}{1-\tau(E)}
$$

We have already shown the behavior of the transmission coefficient as a function of the energy in the previous section, as obtained by means of the transfer matrix formalism. ${ }^{14}$ The calculation of the Landauer conductance is then straightforward for any value of the parameters using the same approach. A typical example of the results is shown in Fig. 2 for the same values of the parameters as in Fig. 1. For a single realization, Fig. 2(a) shows that the detailed structure of the energy spectrum naturally determines the finer details of the conductance pattern at zero temperature. Thus, the noisy aspect of the curve. However, by comparing it to the average plot-
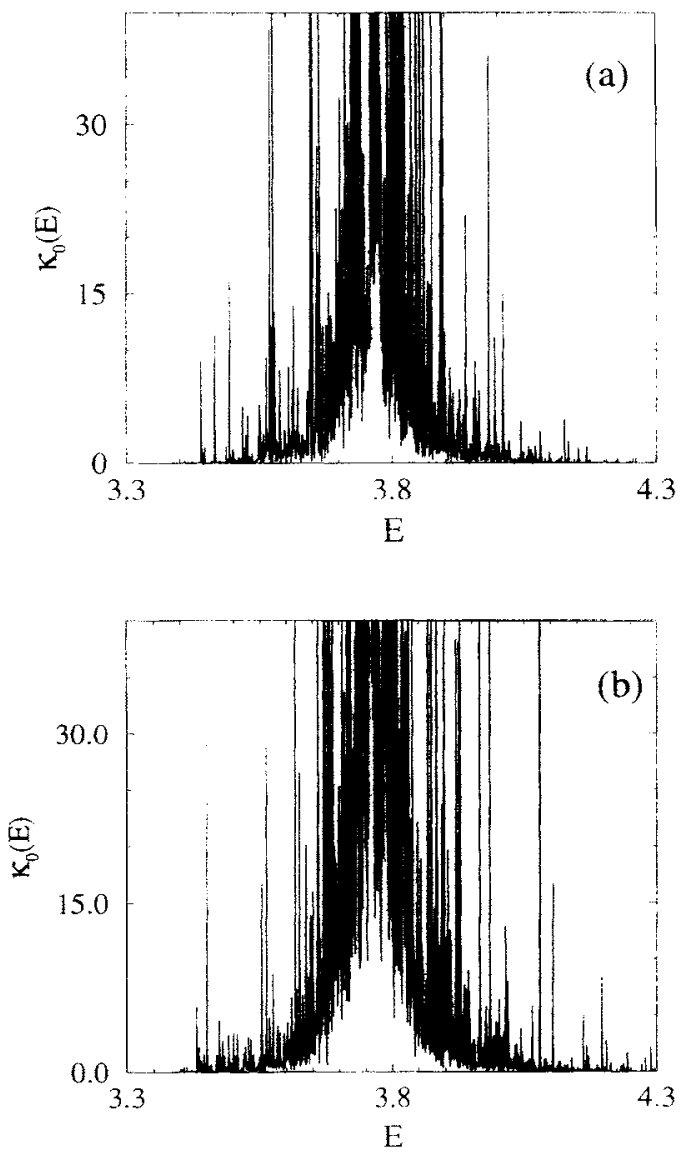

FIG. 2. Landauer conductance at zero temperature for a CDSL with the same parameters as in Fig. 1. (a) A single realization and (b) an average over 100 realizations.

ted in Fig. 2(b), we realize that the result is the very close to the single realization one (as we mentioned when discussing the transmission coefficient), except for the fact that some particular, realization-dependent conductance spikes are suppressed. Note that the computation involves the ratio of the reflection to the transmission coefficient and this quotient enlarges fluctuations considerably, thus the noisy aspect of Fig. 2(b). Obtaining an smoothing as in Fig. 1 would involve averaging over many more realizations. This is very important and we will take advantage of this fact when we discuss how to measure the width of the extended states band, but we can already assume that this magnitude is of order of the width of the peak of $\kappa_{0}$ and that it can be determined from a single realization, i.e., a single superlattice. Finally, we note that the behavior reported here for the first resonance is equally verified for the subsequent ones, so the discussion is not restricted to this first band which should be merely taken as an example.

\section{Finite-temperature dc conductance}

We now proceed to compute the electrical conductance at any temperature. We have already computed this 
magnitude and its relation to the energy spectrum for $\mathrm{Fi}$ bonacci superlattices in previous works. ${ }^{37,38}$ Those works gave results in agreement with known facts about this kind of superlattices (see discussions in Ref. 37). We can then be confident that our calculation will also be relevant in the physical context we are dealing with. The idea in including temperature effects is twofold. First, we then have a control parameter that can be varied at will and correspondingly, we can have a whole set of measurements. Second, the previous results on very low temperature conductance have a limited range of validity and in order to compare with experiments temperature should be included in our results.

The dimensionless finite temperature conductance can be obtained through the following expression, earlier discussed in detail by Engquist and Anderson: ${ }^{39}$

$$
\kappa(T, \mu)=\frac{\int\left(-\frac{\partial n}{\partial E}\right) \tau(E) d E}{\int\left(-\frac{\partial n}{\partial E}\right)[1-\tau(E)]} d E
$$

where integrations are extended over the allowed bands, $\tau(E)$ is the transmission coefficient, $n$ is the Fermi-Dirac distribution, and $\mu$ denotes the chemical potential of the sample. We have calculated expression (10) numerically using the transmission coefficient as input. We discuss separately the cases of low temperatures and the high temperature limit in the following.

\section{Low temperatures}

A global view of the results is presented in Fig. 3. Again, averages smooth out the realization dependent features and preserve the common structure, namely, the clear peak in the conductance around the resonant energy. We also show for comparison what is obtained in the case when we remove the dimer constraint, i.e., for a purely random superlattice. Taking into account the largely different scales between Figs. 3(a) and 3(c), the uncorrelated random superlattice conductivity is very low for all energies. The small features appearing in the plot are specific of the chosen realization and when one takes averages the final result is a flat, zero plot. We then prove that there should be an enormous increase in conductivity, clearly noticeable through experiments when the dimer constraint is satisfied. We note in passing that the result for the pure random system confirms the validity of our procedure.

In those plots, the results discussed for zero temperature are also included, and it is clear that for not so large temperatures the system exhibit a conductance behavior that reproduces the transmission coefficient of the first band, around which the figure is centered. As we start increasing temperature, the peak lowers and widens, and it is already difficult to appreciate for values of $k T$ around 0.5 (around a $5 \%$ of the perfect lattice bandwidth). It is remarkable that in this high temperature region the conductance is clearly nonzero, to be compared to that of the pure random system. It is not difficult to understand why this is so. For low temperatures, the derivative of the Fermi-Dirac function is very peaked around the chemical potential. Therefore, only when the chemical potential is close to the band of extended states, that is to say, close to the resonance, will there be positive contributions to the conductance, and chemical potentials far from the resonance will show zero conductance. As temperature is increased, the derivative of the Fermi-Dirac function becomes wider, and consequently, it is not necessary to choose a chemical potential close to the resonance; even if it is placed far from it the integrals will include the contribution of the extended states. On the contrary, the peak height decreases because previously, for chemical potentials in the band, the localized states outside were not present in the integration, whereas for larger temperatures they contribute in a negative fashion to the
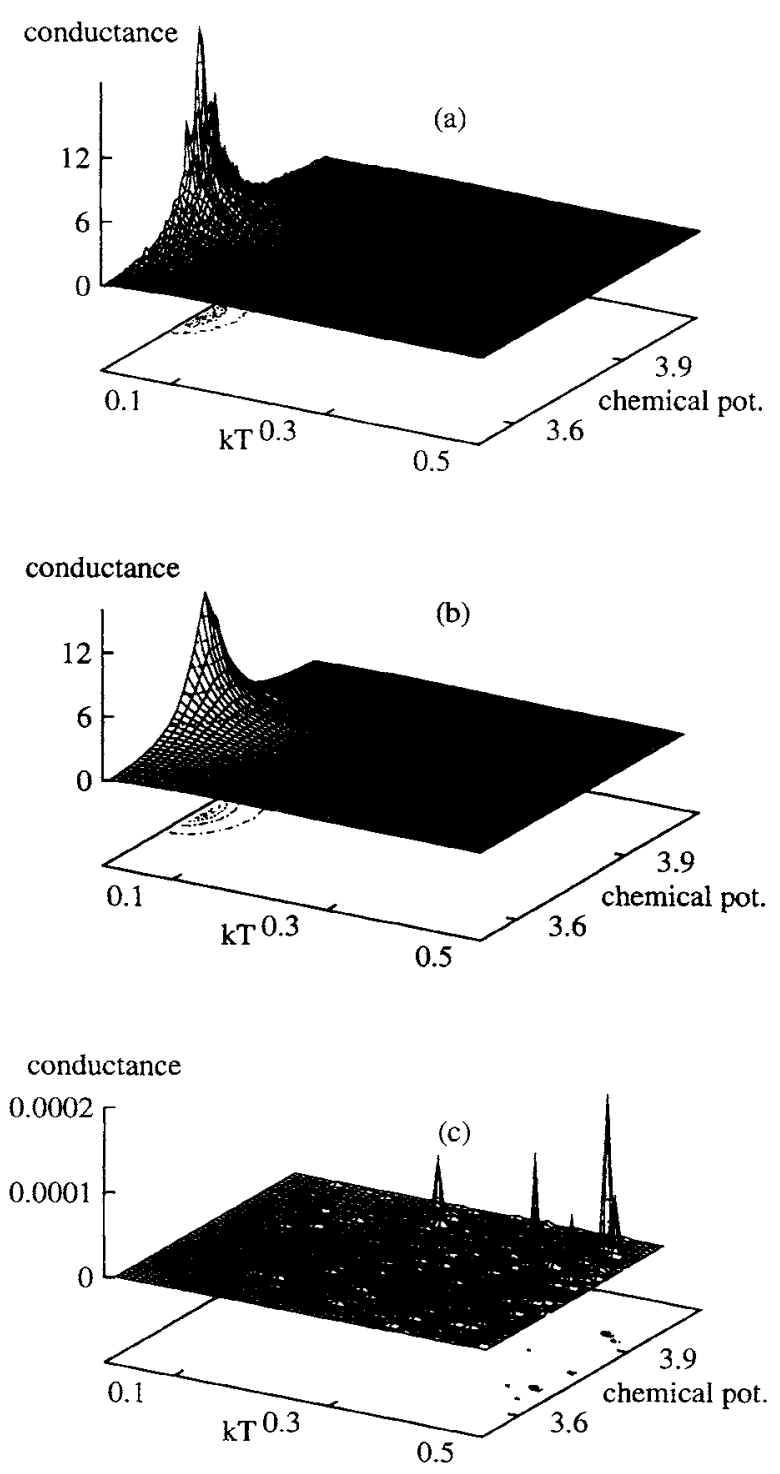

FIG. 3. Finite-temperature conductance as a function of temperature and chemical potential for a CDSL with the same parameters as in Fig. 1. (a) A single realization, (b) an average over 100 realizations, and (c) a single realization without the dimer constraint, i.e., pure randomly disordered lattice. Note the very different vertical scales. 
conductance properties, and they are weighted more in the integration. The behavior we show in Fig. 3 coincides then with the intuitive expectations.

At this point, we recall the analytical approximation we presented in Fig. 1 for the transmission coefficient. An evident way to check whether this magnitude actually behaves in average in such a smooth manner is to use the fitted expression in Eq. (10) and compute again the conductance. As is depicted in Fig. 4, the agreement is very good between both procedures. We can then assume that our fitting is a correct description of the transmission coefficient dependence on the temperature even for each realization, provided that we are not interested in the particular, noisy characteristics of them. This is so because Fig. 3(b) has been computed by generating realizations of the model, computing the transmission coefficient and the conductance, and after that averaging this last quantity, whereas the theoretical calculation in Fig. 4 uses the analytic expression for the transmission coefficient only one, and no averages are involved. That is why we may conclude that the analytic expression can be used for a typical realization. Comparison with transmission coefficients plots for a single realization is also satisfactory. Finally, we point out that the main contribution to the conductance comes from the extended states close to the resonance. This we checked by using a simple parabolic fit to the transmission coefficient, neglecting the tails. The result is again very similar to Fig. 3 , reinforcing our previous conclusion.

\section{High temperature limit}

Figure 3 indicates that the conductance curve rapidly saturate towards a value $\kappa_{\infty}$, independent of the chemical potential, when the temperature reaches a value of the order of $0.5 k T$. The reason why the asymptotic dependence on the temperature is independent of $\mu$ is easy to understand, and we have already discussed it in Ref. 37. The idea goes as follows: Assume we are in the high temperature regime. In this regime, all electrons contribute to vertical transport. Now, define $\epsilon \equiv E-\mu$; for high temperatures $\beta \epsilon<1$ and we can expand the Fermi-Dirac derivative as

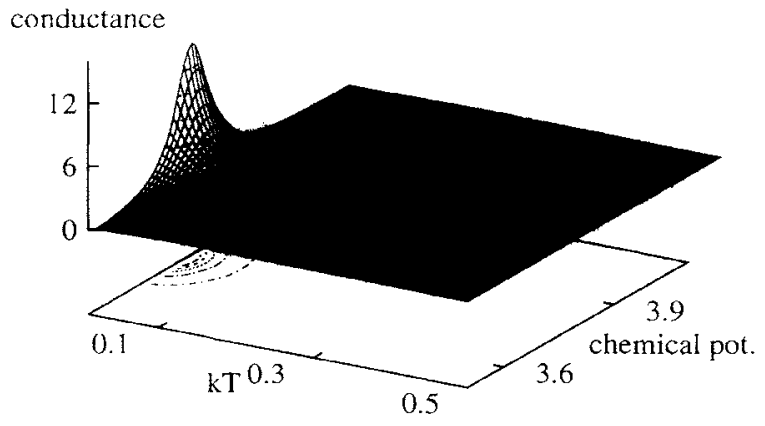

FIG. 4. Finite-temperature conductance obtained using the fitting with Moyal and Gaussian functions in Fig. 1 in Eq. (10).

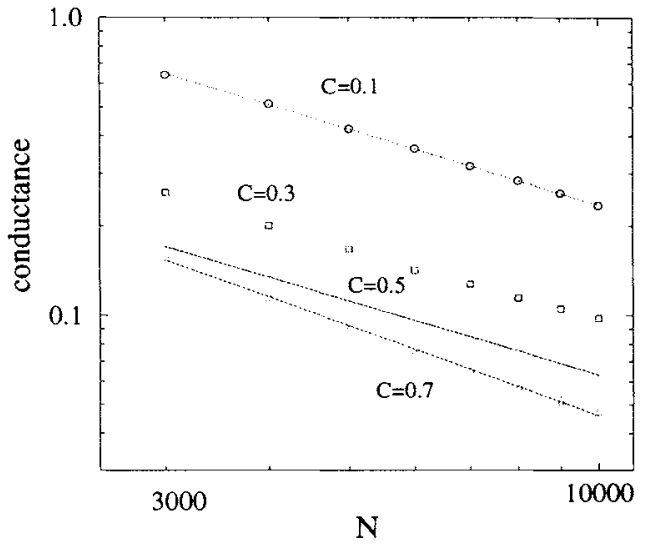

FIG. 5. Dependence of the high temperature conductance on the sample length for several values of the concentration showing their power-law behavior. Solid lines are least squares fits with slopes of order of -0.85 .

$$
-\frac{\partial n}{\partial E} \simeq \frac{1}{4}+O\left[(\beta \epsilon)^{2}\right]
$$

which inserted into the general formula (10) yields

$$
\kappa_{\infty} \simeq \frac{\int \tau(E) d E}{\int[1-\tau(E)]} d E,
$$

an expression where the chemical potential has disappeared, and the only dependence is on the number of $\delta$ functions, their strengths, and their concentrations, all these quantities entering through the transmission coefficient. In fact, we have checked that $\kappa_{\infty}$ scales with the number of scatterers as a power law, with exponent depending on the dimer concentration as we show in Fig. 5. Similar results are obtained by changing the strengths of the $\delta$ functions. On the other hand, from these same reasonings it can be induced that the vanishing of the high temperature conductance of the pure random system is due to the fact that the transmission coefficient is always close to zero, and there are no extended states. Once again we see that the dimer structure gives rise to largely different features as compared to the random one.

\section{DISCUSSION AND CONCLUSIONS}

After reporting all our study of the finite-temperature dc conductance of CDSL's, we are now in the best position to discuss what are the means to obtain the characteristics of the band of extended states from experimental measurements. We mentioned in the motivation that we intended to find the position of the bands, for instance, the first one and their width. We begin with the system that should be used: We believe that any of the experimental setups used in previous works, short period $\mathrm{GaAs} / \mathrm{Ga}_{1-x} \mathrm{Al}_{x} \mathrm{As}$ (Refs. 21-25) or SiGe/Si (Ref. 26) are suitable devices for the kind of measurementes we proposed, provided that they are built with the dimer con- 
straint. Electronic transport through the so built CDSL can be measured either by techniques that employ magnetic or electric fields, ${ }^{40}$ or by all-optical procedures, ${ }^{41}$ or by a combination of both that helps avoid the intrinsic experimental problems of each of them.

Let us now turn to the measurements themselves. The first quantity we have to determine is the position of the extended band of states. The way to do that is to prepare several superlattices with different chemical potentials. This could be achieved by varying doping concentration or pressure. These samples would have different chemical potential, but as far as the nature of the layers forming the dimer is not changed, the position of the bands must be the same. Therefore, the particular sample for which a maximum of the conductivity were reached would be that with the chemical potential closer to the required band. Even if the first band is well below the Fermi level, as there are infinite other bands, some of them would be reached and a rapid increase in conductivity should be noticeable. Further, higher order bands are wider, so they should be even easier to detect. The question remains as to what is the appropriate range of temperatures to look at, because if temperature is too high no maximum should be detected. As we have already mentioned, this limit is reached at about $k T$ of order the of $5 \%$ of the perfect lattice bandwidth. In typical superlattices this width is close to $100 \mathrm{meV}$ (say). Thus high temperature limit means that $k T \sim 5 \mathrm{meV}$, that is, $T$ around liquid nitrogen temperature. Therefore, the marked peak in the dc conductance should be clearly observable at temperatures close to $5-10 \mathrm{~K}$ in most superlattices with different values of the chemical potential. The practical implications of this result is twofold. First, the range of temperature is physically realizable and second, electron-phonon interaction can be neglected, as we have assumed. On the other hand, these comments are also related to the filter application we mentioned in Sec. III $\mathrm{A}$, as it is evident that high temperatures will obscure this behavior. In this respect, we can estimate the temperatures at which the loss of selectivity makes the device useless using the above ideas; as we have said, the peak is clearly observable below $k T \simeq 0.5$. This can also be seen from the fact that for such low temperatures the derivative of the Fermi distribution has a width of the order of $k T$, which roughly coincides with the width of $\tau(E)$ and $\kappa(E)$. Therefore, dimer-based filters will be efficient in the range of liquid nitrogen temperature and below. Of course, this applies only to the set of parameters we are dealing with, and changing the strength of the dimers $\lambda^{\prime}$ as well as that of the host lattice $\lambda$ may allow us to improve this quite conservative bound.

So, we may suppose that we have hit a band of extended states, and that we know approximately the location of the center. The next step is to perform a number of measurements, for some values of the chemical potential at different temperatures. If the chemical potential does not need to be varied much the use of CDSL's with different dimer concentrations could be considered as well. If we were able to measure the conductance at zero temperature, we would have a portrait of the transmission coefficient itself. However, this is not possible,

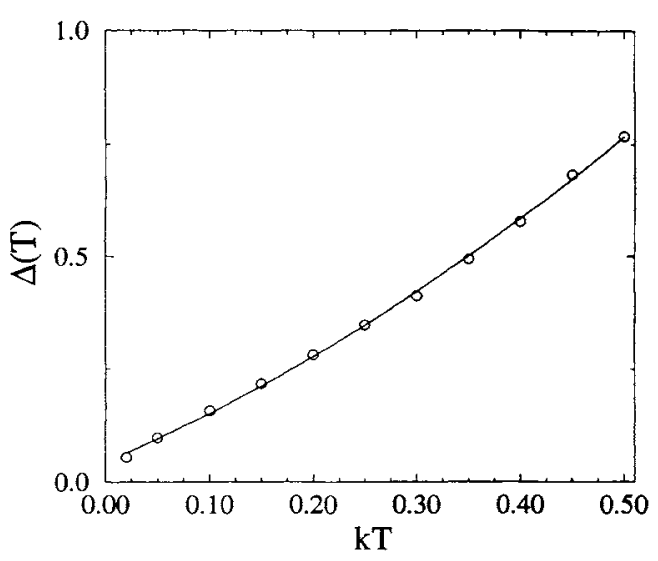

FIG. 6. Dependence of the width of the conductance peak on the temperature as given by Gaussian fits. System parameters are as in Fig. 1.

but what we can always do is measure as close to zero as to have an idea of what the shape of the transmission coefficient is. A more quantitative way to do this is the following: Take a series of measurements of the conductance for different values of temperature. For each of the so obtained profiles (actually sections of Fig. 3) one can compute its width by a number of means (and also depending on the definition of width itself). For instance, what one can do is to fit Gaussians to the experimental profiles, if it is not desired to use the more sophisticated function mentioned in Sec. II. That would provide the width $\Delta$ as a function of $T$. We carried out this in our model and the result is shown in Fig. 6. In this plot we may note that the width behaves as $\Delta(T)=a_{0}+a_{1} T+a_{2} T^{2}$, with $a_{0} \sim 0.05, a_{1} \sim 1$ and $a_{2} \sim 1$. The value of the width of the band of extended states is then simply $a_{0}$ (i.e., the fitting evaluated at $T=0$ ). We have thus provided a means to estimate the width of this band for CDSL's.

Finally, to ensure consistency of all the procedure, and also to help choose the better regime to work on, we can also examine how the peak width depends on the

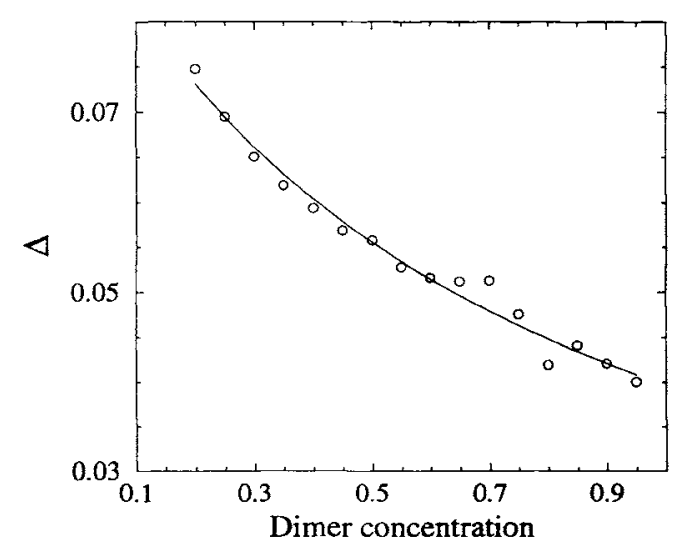

FIG. 7. Dependence of the width of the conductance peak at $k T=0.02$ on the dimer concentration as given by Gaussian fits. System parameters are as in Fig. 1. 


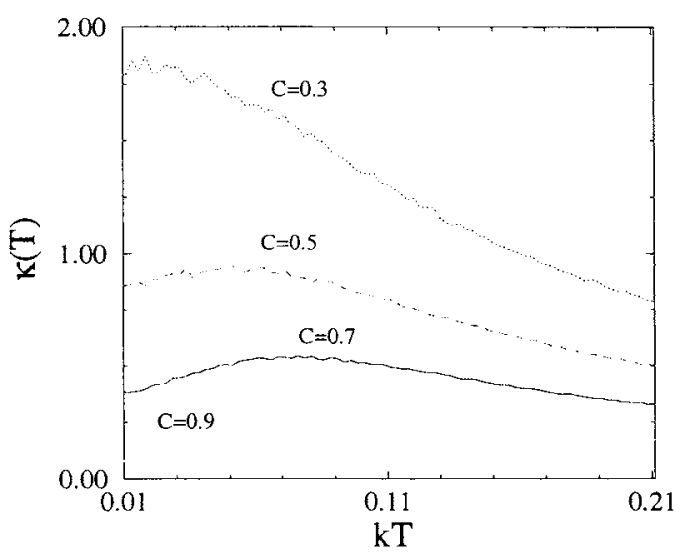

FIG. 8. Conductance as a function of temperature for different concentrations. Chemical potential is always placed at the middle of the band of extended states.

concentration of dimers and also how it behaves with temperature for a given concentration. The results are shown in Figs. 7 and 8. Figure 7 exhibits a dependence of the width $\Delta$ on the dimer concentration basically as $c^{-1}$. This makes sense, because for concentration almost zero the superlattice would be practically perfect, and the good properties of the dimers would leave most states unscattered. If we now look at the temperature where the maximum of the conductance is achieved, we see that it moves towards higher temperatures (see Fig. 8), with a functional form which is again roughly $c^{-1}$. This may facilitate working on higher temperatures if needed.

In conclusion, we have studied finite-temperature conductance of the CRDM and have shown how this study leads to specific predictions that may be measured on actual superlattices. If performed, those experiments would validate (or discard) all the recent claims that correlation induces the appearance of bands of extended states in spite of the localization effects of disorder. Aside from our suggestions above, of already studied quantum well superlattices, it is most interesting to note that recent results on a single $\mathrm{Si}$ cell with double $\delta$ doping ${ }^{42}$ have been reported, and they exhibit a large increase of electron mobility in this kind of structure as compared to single or homogeneously doped structures. Although our analysis may not apply straightforwardly to this measurement, it is tempting to suggest that at the roots of the behavior may be the dimer resonance effect, at least partially. On the other hand, $\delta$ doped structures might be even more suitable to this kind of experiments and fit better the theoretical model we have been discussing. We hope that this work stimulates experimental efforts in this direction.

\section{ACKNOWLEDGMENTS}

It is with great pleasure that we thank collaboration and illuminating conversations with Enrique Maciá. A.S. is also thankful to Alan Bishop for warm hospitality at Los Alamos National Laboratory where this paper was written in part. Work at Leganés is supported by the Dirección General de Investigación Científica y Técnica (Spain) through project PB92-0248, and by the European Union Human Capital and Mobility Programme through Contract No. ERBCHRXCT930413. Work at Madrid is supported by Universidad Complutense through Project No. PR161/93-4811.
${ }^{1}$ J. C. Flores, J. Phys. Condens. Matter 1, 8471 (1989).

${ }^{2}$ D. H. Dunlap, H.-L. Wu, and P. Phillips, Phys. Rev. Lett. 65, 88 (1990).

${ }^{3}$ H.-L. Wu and P. Phillips, J. Chem. Phys. 93, 7369 (1990).

${ }^{4}$ H.-L. Wu and P. Phillips, Phys. Rev. Lett. 66, 1366 (1991).

${ }^{5}$ P. Phillips and H.-L. Wu, Science 252, 1805 (1991).

${ }^{6}$ A. Bovier, J. Phys. A 25, 1021 (1992).

${ }^{7}$ H.-L. Wu, W. Goff, and P. Phillips, Phys. Rev. B 45, 1623 (1992).

${ }^{8}$ S. N. Evangelou and D. E. Katsanos, Phys. Lett. A 164, 456 (1992).

${ }^{9}$ P. K. Datta, D. Giri, and K. Kundu, Phys. Rev. B 47, 10727 (1993).

${ }^{10}$ S. N. Evangelou and A. Z. Wang, Phys. Rev. B 47, 13126 (1993).

${ }^{11}$ F. Domínguez-Adame, E. Maciá, and A. Sánchez, Phys. Rev. B 48, 6054 (1993).

12 J. C. Flores and M. Hilke, J. Phys. A 26, L1255 (1993).

${ }^{13}$ A. Sánchez and F. Domínguez-Adame, J. Phys. A 27, 3725 (1994).

${ }^{14}$ A. Sánchez, E. Maciá, and F. Domínguez-Adame, Phys. Rev. B 49, 147 (1994); 49, 15 428(E) (1994).

${ }^{15} \mathrm{~F}$. Domínguez-Adame, B. Méndez, A. Sánchez, and E. Maciá, Phys. Rev. B 49, 3839 (1994).
${ }^{16}$ F. Domínguez-Adame, E. Maciá, and A. Sánchez, Phys. Rev. B 50, 6453 (1994).

${ }^{17}$ R. Brito, F. Domínguez-Adame, and A. Sánchez (unpublished).

${ }^{18}$ A. Sánchez, F. Domínguez-Adame, and E. Maciá, Phys. Rev. B (to be published).

${ }^{19}$ J. M. Ziman, Models of Disorder (Cambridge University Press, London, 1979).

${ }^{20}$ R. Merlin, K. Bajema, R. Clarke, F.-Y. Juang, and P. K. Battacharya, Phys. Rev. Lett. 55, 1768 (1985); J. Todd, R. Merlin, R. Clarke, K. M. Mohanty, and J. D. Axe, ibid. 57, 1157 (1986).

${ }^{21}$ A. Chomette, B. Deveaud, A. Regreny, and G. Bastard, Phys. Rev. Lett. 57, 1464 (1986).

${ }^{22}$ A. Sasaki, M. Kasu, T. Yamamoto, and S. Noda, Jpn. J. Appl. Phys. 28, L1249 (1989).

${ }^{23}$ M. Kasu, T. Yamamoto, S. Noda, and A. Sasaki, Appl. Phys. Lett. 59, 800 (1991), and references therein.

${ }^{24}$ E. Tuncel and L. Pavesi, Philos. Mag. B 65, 213 (1992).

${ }^{25}$ D. J. Arent et al., Phys. Rev. B 49, 11173 (1994).

${ }^{26}$ A. Wakahara, T. Hasegawa, K. Kuramoto, K. V. Vong, and A. Sasaki, Appl. Phys. Lett. 64, 1850 (1994).

${ }^{27}$ R. de L. Kronig and W. G. Penney, Proc. R. Soc. London Ser. A 130, 499 (1931). 
${ }^{28}$ M. Jaros, Physics and Application of Semiconductor Microstructures (Clarendon Press, Oxford, 1989).

${ }^{29}$ See, e.g., E. Lieb and D. C. Mattis, Mathematical Physics in One Dimension (Academic Press, New York, 1966).

${ }^{30}$ Y. Tanaka and M. Tsukada, Phys. Rev. B 40, 4482 (1989).

${ }^{31}$ G. J. Clerk and B. H. J. McKellar, Phys. Rev. C 41, 1198 (1990).

${ }^{32}$ F. Domínguez-Adame, J. Phys. Condens. Matter 1, 109 (1989).

${ }^{33}$ P. Erdós and R. C. Herndon, Helv. Phys. Acta 50, 513 (1977).

${ }^{34}$ B. Méndez, F. Domínguez-Adame, and E. Maciá, J. Phys. A 26, 171 (1993).

35 J. E. Moyal, Philos. Mag. 46, 263 (1955); P. V. Vavilov, Zh. Eksp. Teor. Fiz. 32, 920 (1957) [Sov. Phys. JETP 5, 749 (1957)].

${ }^{36}$ R. Landauer, IBM J. Res. Dev. 1, 223 (1957).
${ }^{37}$ E. Maciá, F. Domínguez-Adame, and A. Sánchez, Phys. Rev. B 49, 9503 (1994).

${ }^{38}$ E. Maciá, F. Dominguez-Adame, and A. Sánchez, Phys. Rev. E 50, 679 (1994).

${ }^{39}$ H. L. Engquist and P. W. Anderson, Phys. Rev. B 37, 1097 (1988).

40 J. F. Palmier, C. Minot, J. L. Lievin, F. Alexandre, J. C. Harmand, J. Dangla, C. Dubon-Chevallier, and D. Ankri, Appl. Phys. Lett. 49, 1260 (1986); T. Duffield, R. Bhat, M. Koza, F. de Rosa, D. M. Hwang, P. Grabbe, and S. J. Allen, Jr., Phys. Rev. Lett. 56, 2724 (1990).

${ }^{41}$ T. Amand, J. Barrau, X. Marie, N. Lauret, B. Dareys, M. Brosseau, and F. Laruelle, Phys. Rev. B 47, 7155 (1993), and references therein.

${ }^{42}$ H. H. Radamson, M. R. Sardela, Jr., O. Nur, M. Willander, B. E. Sernelius, W.-X. Ni, and G. V. Hansson, Appl. Phys. Lett. 64, 1842 (1994). 

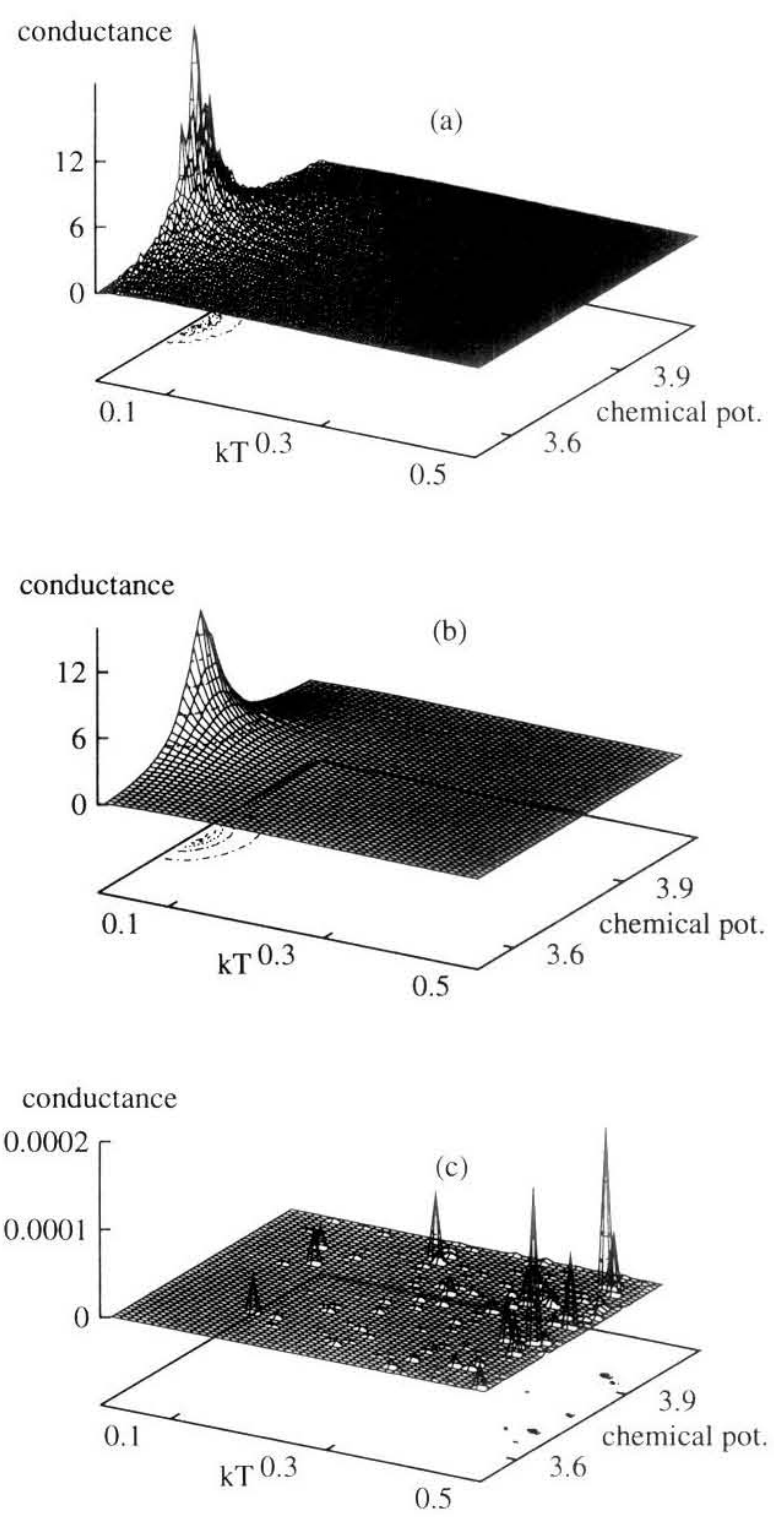

FIG. 3. Finite-temperature conductance as a function of temperature and chemical potential for a CDSL with the same parameters as in Fig. 1. (a) A single realization, (b) an average over 100 realizations, and (c) a single realization without the dimer constraint, i.e., pure randomly disordered lattice. Note the very different vertical scales. 


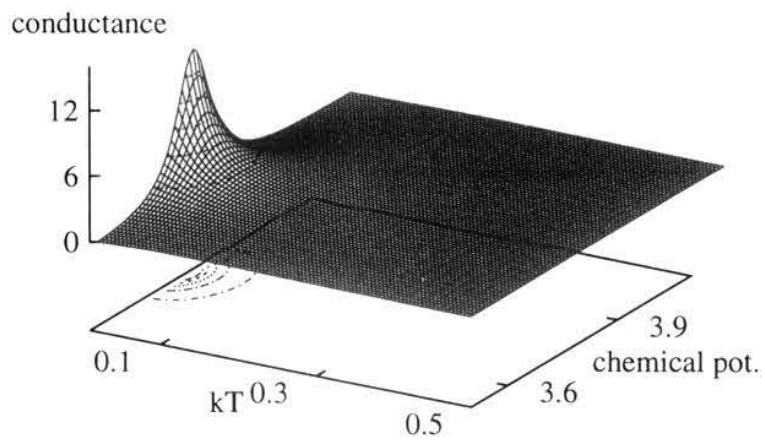

FIG. 4. Finite-temperature conductance obtained using the fitting with Moyal and Gaussian functions in Fig. 1 in Eq. (10). 